\title{
Effect of fluid static pressure on the immediate postbuckling behavior of heavy tubular columns
}

\section{THEODORE KOKKINIS}

\author{
Department of Mechanical and Environmental Engineering, University of California, \\ Santa Barbara, CA 93106, USA
}

\section{MICHAEL M. BERNITSAS}

Department of Naval Architecture and Marine Engineering, The University of Michigan, Ann Arbor, MI 48109-2145, USA

Key Words: marine risers, fluid pressure, postbuckling behavior, imperfection sensitivity.

\section{INTRODUCTION}

Heavy tubular columns are prone to global static instability under the combined action of their weight, tension or compression exerted at their upper end and internal and external static pressure forces due to fluids in gravity field. The initial postbuckling behavior of such column, after static instability occurs, affects the sensitivity of the buckling loads to lateral load and initial deformation, the magnitude of the prebuckling deformation and the shape of the equilibrium path of the laterally loaded column. The latter determines the relationship between slope at the column's lower end and applied top tension.

Koiter ${ }^{1}$ developed a theory for the initial postbuckling behavior of structures subject to conservative loading. According to this theory an analysis of the potential energy of the structure in the neighborhood of the bifurcation point under the assumption that the deformation has the form of the buckling mode shape can determine the initial slope and curvature of the secondary equilibrium path. ${ }^{2}$ On the basis of slope and curvature the effect of imperfections can be judged.

The buckling of heavy columns has received a lot of attention in the past. Greenhill ${ }^{3}$ found the critical density or height of a uniformly weighted column. Willers ${ }^{4}$ studied heavy columns with end load and attempted an asymptotic solution for infinitely long columns. Biezeno and $\mathrm{Koch}^{5}$ studied the buckling of a submerged tube with closed ends. Huang and Dareing ${ }^{6,7}$ considered the buckling of long vertical pipes subject to external hydrostatic pressure. Bernitsas and Kokkinis ${ }^{8}$ studied the elastic stability of marine risers, subject to internal and external fluid static pressure, and proved that heavy columns may buckle globally due to internal pressure while they are in tension over their entire length. ' In addition they determined the stability boundaries for very long columns using asymptotic and quasiasymptotic techniques. ${ }^{19}$ All the aforementioned papers deal with the derivation of stability boundaries and buckling loads.

The analytical solution of the problem of the postbuckling behavior of a weightless column subject to end

Accepted May 1986. Discussion closes September 1987. load (elastica) in terms of elliptic functions is well estab. lished. ${ }^{10}$ This same problem has been tackled using various approximate techniques by Sewell, ${ }^{11}$ Croll, ${ }^{12}$ Thompson and Hunt $^{13}$ and Berkey and Friedman. ${ }^{14}$ Plaut $^{15}$ considered the postbuckling of a cantilever subject to two independent compressive loads. Wang ${ }^{16}$ found postbuckling shapes for suspended heavy columns with end load using a shooting technique to integrate the nonlinear equation of equilibrium. Kokkinis and Bernitsas ${ }^{17}$ used a finite element technique to determine the initial portion of the secondary equilibrium path of heavy columns and risers. The numerical results derived indicated the possibility of unstable postbuckling behavior. The present work was undertaken in order to verify these results using an analytical method and to derive conditions for the occurrence of unstable behavior.

The fluid static pressure forces acting on heavy columns are nonconservative, therefore Koiter's method of analyzing the potential energy is inapplicable in this case. An analogous technique is adopted starting from the nonlinear equation of equilibrium. Inequality conditions for unstable initial postbuckling behavior are developed between the various dimensionless parameters of the problem. These conditions can be used to determine whether the immediate postbuckling behavior of a column if stable or unstable.

The present work was initiated as part of the study of the elastic stability of marine risers. The results derived are general enough to be applicable to many types of hydraulic columns. They are, however, primarily applicable to marine risers. A marine drilling riser is treated in a numerical example. It is shown that the the initial postbuckling behavior of the riser is unstable for relatively low values of the drilling mud density. As a result the riser will buckle at a top tension higher than the theoretically predicted if it has initial deformation and is loaded laterally as is usually the case in practice. This also results in greater prebuckling deformation and particularly at the bottom.

\section{MATHEMATICAL MODEL}

The model employed to study the initial postbuckling behavior of tubular columns consists of a nonlinear integrodifferential equation governing the slope of the centerline 
and two rotation conditions at the ends. It is based on the following assumptions:

1. Shear deformations are small. Consequently, the column can be modelled as an Euler-Bernoulli beam.

2. The column material is isotropic, homogeneous and linearly elastic.

3. Torsional deformation and extension of the column are small and can be neglected.

4. The column ends are open.

5. Strains in the column are small, although slopes and deflections may be large.

6. The deformation of the column is planar.

The lower end of the column is assumed to be fixed for translation in any direction. The upper end is subject to a vertical tension/compression, while its horizontal translation is resisted by a linear spring.

The governing nonlinear integrodifferential equation derived in the Appendix is:

$$
\begin{aligned}
& \frac{\mathrm{d}}{\mathrm{d} s}\left(E I \frac{\mathrm{d} \theta}{\mathrm{d} s}\right)-\left[-\int_{s}^{L} w_{e} \mathrm{~d} \sigma+T_{t, a}\right] \sin \theta \\
& \quad-T_{t, f} \sin [\theta-\theta(L)]-k_{t} x(L) \cos \theta=0
\end{aligned}
$$

where $\theta$ is the slope of the column centerline, $L$ is the length, $E I$ is the bending rigidity, $w_{e}$ is the effective weight per unit length, $T_{t, a}$ is the applied tension at the top, $k_{t}$ is the linear spring stiffness at the top,

$$
T_{t, f}=b_{w}(L)\left[h_{w}-z(L)\right]-w_{m}(L)\left[h_{m}-z(L)\right]
$$

is the fictitious fluidic tension at the top and

$$
x(L)=\int_{0}^{L} \sin \theta \mathrm{d} s \text { and } z(L)=\int_{0}^{L} \cos \theta \mathrm{d} s
$$

are the horizontal and vertical displacements of the upper end. Furthermore $b_{w}$ is the weight of the displaced fluid and $w_{m}$ the weight of the contained fluid per unit length. Also $h_{w}$ is the water depth and $h_{m}$ the free surface ordinate of the contained fluid. The rotation condition at either end can be either hinged

$$
\left.\frac{\mathrm{d} \theta}{\mathrm{d} s}\right|_{s=0, L}=0
$$

or clamped

$$
\left.\theta\right|_{s=0, L}=0
$$

For small slopes and deflections the governing equation can be linearised

$$
\begin{aligned}
& \frac{\mathrm{d}}{\mathrm{d} s}\left(E I \frac{\mathrm{d} \theta}{\mathrm{d} s}\right)-\left[-\int_{s}^{L} w_{e} \mathrm{~d} \sigma+T_{t, a}\right] \theta \\
& -\left(T_{t, f}\right)_{l}[\theta-\theta(L)]-k_{t} \int_{0}^{L} \theta \mathrm{d} \sigma=0
\end{aligned}
$$

where $\left(T_{t, f}\right)_{l}$ is the linearised expression for the fictitious fluidic tension at the top

$$
\left(T_{t, f}\right)_{i}=b_{w}(L)\left(h_{w}-L\right)-w_{m}(L)\left(h_{m}-L\right)
$$

In this linearised equation (5) we can substitute $x$, the co-ordinate along the undeformed axis of the column, for $s$, the co-ordinate along the deformed axis of the column, as the independent variable, because of the assumption of small slopes and deflections. This is not possible for the general equation (1) which is derived for large slopes and deflections.

The linearised equation (5) together with boundary conditions (4a) and (4b) constitutes an eigenvalue problem, which in general is nonselfadjoint. An eigenvalue problem consisting of the linear homogeneous differential equation $L(\theta)=0$ and associated linear homogeneous boundary conditions is self-adjoint, according to the definition given by Bolotin, ${ }^{18}$ if

$$
\int_{0}^{L}[L(\theta) \tilde{\theta}-L(\vec{\theta}) \theta] \mathrm{d} s=0
$$

where $\theta$ and $\bar{\theta}$ satisfy the boundary conditions. In the case of the heavy tubular column the expression in equation (7) is

$$
\int_{0}^{L}[L(\theta) \bar{\theta}-L(\bar{\theta}) \theta] \mathrm{d} s=-\left.\left(T_{t, f}\right)_{l}(\theta \bar{x}-\bar{\theta} x)\right|_{s=L}
$$

This expression is in general nonzero and thus the riser eigenvalue problem is nonselfadjoint. A nonselfadjoint eigenvalue problem corresponds to a physical system with nonconservative forces. The nonconservative forces in the riser system are the fluid pressure forces, as demonstrated by equation ( 8 ). The expression in equation (8) becomes zero and therefore the eigenvalue problem becomes selfadjoint if the linearised expression for the fluidic tension at the top is zero. A sufficient condition for this is

$$
h_{m}=h_{w}=L
$$

This assumption is adopted in this work because it is approximately valid for many riser installations. Although under this assumption the eigenvalue problem becomes selfadjoint, the nonconservative forces from fluid pressure are still present and manifest themselves in the nonlinear equation (1). The linearised differential equation under assumption (9) becomes

$$
\frac{\mathrm{d}}{\mathrm{d} s}\left(E I \frac{\mathrm{d} \theta}{\mathrm{d} s}\right)-\left[-\int_{s}^{L} w_{e} \mathrm{~d} \sigma+T_{t, a}\right] \theta-k_{t} \int_{0}^{L} \theta \mathrm{d} \sigma=0
$$

The solution of the eigenvalue problem consisting of (10) and boundary conditions (4a) and/or (4b) yields the critical top tension $\left(T_{t, a}\right)_{c}$ and the buckling mode shape $\Theta_{c}(s)$.

\section{IMMEDIATE POSTBUCKLING ANALYSIS}

The analysis of the immediate postbuckling behavior of the column is carried out by solving the nonlinear equation (1) approximately using the assumption that in the neighborhood of the bifurcation point the deformation is of the form of the buckling mode, namely that

$$
\Theta(s)=\epsilon \Theta_{c}(s)
$$

where $\epsilon$ is small. 
Let us introduce the following notation

$$
\int_{s}^{L} w_{e} \mathrm{~d} \sigma=w_{e, o}(s)
$$

Then applying (11) to the governing equation (1) we get

$$
\begin{aligned}
& \frac{\mathrm{d}}{\mathrm{d} s}\left[E I \frac{\mathrm{d}\left(\epsilon \Theta_{c}\right)}{\mathrm{d} s}\right]-\left[T_{t, a}-w_{e, o}(s)\right] \sin \left(\epsilon \Theta_{c}\right) \\
& \quad-\left[b_{w}(L)-w_{m}(L)\right]\left[L-\int_{0}^{L} \cos \left(\epsilon \Theta_{c}\right) \mathrm{d} \sigma\right] \\
& \quad \times \sin \left\{\epsilon\left[\theta_{c}-\theta_{c}(L)\right]\right\}-k_{t}\left[\int_{0}^{L} \sin \left(\epsilon \Theta_{c}\right) \mathrm{d} \sigma\right] \cos \left(\epsilon \Theta_{c}\right) \\
& \quad=E(s)
\end{aligned}
$$

A scalar equation for $\epsilon$ is obtained by demanding that the global error be zero with weighting function $\Theta_{c}(s)$

$$
\int_{0}^{L} E(s) \Theta_{c}(s) \mathrm{d} s=f\left(T_{t, a}, \epsilon\right)=0
$$

This is a Galerkin-type solution with one term in the Galerkin series. Equation (14) can be regarded as an implicit equation of the secondary equilibrium path, which is exact at the bifurcation point and approximate away from it.

After introducing Taylor expansions of the sine and cosine functions and further manipulation, equation (14) becomes

$$
T_{t, a}=\frac{A_{0}-\epsilon^{2} A_{2}+0\left(\epsilon^{4}\right)}{B_{0}-\epsilon^{2} B_{2}+0\left(\epsilon^{4}\right)}
$$

where

$$
\begin{aligned}
A_{0}= & -\int_{0}^{L} E I\left(\frac{\mathrm{d} \Theta_{c}}{\mathrm{~d} s}\right)^{2} \mathrm{~d} s+\int_{0}^{L} w_{e, o} \Theta_{c}^{2} \mathrm{~d} s-k_{T}\left(\int_{0}^{L} \Theta_{c} \mathrm{~d} s\right)^{2} \\
A_{2}= & \frac{1}{6} \int_{0}^{L} w_{e, o} \Theta_{c}^{4} \mathrm{~d} s+\frac{1}{2}\left[b_{w}(L)-w_{m}(L)\right] \\
& \times\left(\int_{0}^{L} \Theta_{c}^{2} \mathrm{~d} s\right)\left\{\int_{0}^{L}\left[\Theta_{c}-\Theta_{c}(L)\right] \Theta_{c} \mathrm{~d} s\right\} \\
& -\frac{2}{3} k_{t}\left(\int_{0}^{L} \Theta_{c} \mathrm{~d} s\right)\left(\int_{0}^{L} \Theta_{c}^{3} \mathrm{~d} s\right) \\
B_{0}= & \int_{0}^{L} \Theta_{c}^{2} \mathrm{~d} s
\end{aligned}
$$

and

$$
B_{2}=\frac{1}{6} \int_{0}^{L} \Theta_{c}^{4} \mathrm{~d} s
$$

$$
\text { As } \epsilon \rightarrow 0,(15) \text { becomes }
$$

$$
\begin{aligned}
\lim _{e \rightarrow 0} T_{t, a}= & \frac{A_{0}}{B_{2}} \\
= & {\left[-\int_{0}^{L} E r\left(\frac{\mathrm{d} \Theta_{c}}{\mathrm{~d} s}\right)^{2} \mathrm{~d} s+\int_{0}^{L} w_{e, o} \Theta_{c}^{2} \mathrm{~d} s\right.} \\
& \left.-k_{t}\left(\int_{0}^{L} \Theta_{c} \mathrm{~d} s\right)^{2}\right] / \int_{0}^{L} \Theta_{c}^{2} \mathrm{~d} s=\left(T_{t, a}\right)_{c}
\end{aligned}
$$

This can be verified by multiplying equation (10) by $\Theta_{c}$ and integrating over the domain.

The column is stable if the applied tension is greater than the critical

$$
T_{t, a}>\left(T_{t, a}\right)_{c}
$$

and becomes unstable if the applied tension is decreased below the critical. Bifurcation of the equilibrium path occurs at

$$
T_{t, a}=\left(T_{t, a}\right)_{c}
$$

After bifurcation the column follows the secondary equilibrium path, which near the bifurcation point has slope

$$
\frac{\mathrm{d} T_{t, a}}{\mathrm{~d} e}=\frac{-2 \epsilon\left(A_{2} B_{0}-A_{0} B_{2}\right)+0\left(\epsilon^{3}\right)}{B_{0}^{2}+0\left(\epsilon^{2}\right)}
$$

At the bifurcation point itself

$$
\lim _{\epsilon \rightarrow 0} \frac{\mathrm{d} T_{t, a}}{\mathrm{~d} \epsilon}=0
$$

as expected for a symmetric structure. Thus the stability of the column's initial postbuckling behavior is to be judged on the basis of the curvature of the secondary equilibrium path at the bifurcation point. This is

$$
\frac{\mathrm{d}^{2} T_{t, a}}{\mathrm{~d} \epsilon^{2}}=\frac{-2 B_{0}^{2}\left(A_{2} B_{0}-A_{0} B_{2}\right)+0\left(\epsilon^{2}\right)}{B_{0}^{4}+0\left(\epsilon^{2}\right)}
$$

which in the limit becomes

$$
\lim _{\epsilon \rightarrow 0} \frac{\mathrm{d}^{2} T_{t, a}}{\mathrm{~d} \epsilon^{2}}=2 \frac{A_{0} B_{2}-A_{2} B_{0}}{B_{0}^{2}}
$$

If this expression is negative, the equilibrium path curves downward, that is nontrivial equilibrium configurations are possible only for applied tension less than critical. This indicates a stable postbuckling behavior.

If on the other hand this expression is positive, the equilibrium path curves upward and nontrivial equilibrium configurations are possible for applied tension greater than critical. This indicates an unstable postbuckling behavior. If the initial postbuckling behavior is unstable the buckling of an initially deformed and/or laterally loaded column will not occur at the theoretically predicted critical top tension but at a higher tension. The value of this actual buckling tension is dependent on the imperfections and the lateral loads. ${ }^{2}$ This indicates that the buckling loads are sensitive to imperfections. Moreover, in this case prebuckling slopes and deflections are greater than in the case of stable behavior. Specifically, in the case of marine risers the slope at the lower ball joint will be affected. 
Fluid static pressure on immediate postbuckling behavior of heavy tubular columns: T. Kokkinis and M. Bernitsas

The transition from stable to unstable postbuckling behavior occurs when the numerator on the right hand side of equation (26) changes from negative to positive. That is the transition occurs when

$$
A_{0} B_{2}-A_{2} B_{0}=0
$$

Using (16)-(19) in (27) we find that the condition for unstable initial postbuckling behavior can be transformed to equation (28) which is a condition on the weight of the contained fluid per unit length.

$$
\begin{aligned}
& w_{m}(L) \geqslant b_{w}(L)+\frac{1}{3}\left\{\left[\int_{0}^{L} E I\left(\frac{\mathrm{d} \Theta_{c}}{\mathrm{~d} s}\right)^{2} \mathrm{~d} s\right]\left(\int_{0}^{L} \Theta_{c}^{4} \mathrm{~d} s\right)\right. \\
& +\left[\left(\int_{0}^{L} w_{e, o} \Theta_{c}^{4} \mathrm{~d} s\right)\left(\int_{0}^{L} \Theta_{c}^{2} \mathrm{~d} s\right)\right. \\
& -\left(\int_{0}^{L} w_{e, o} \Theta_{c}^{2} \mathrm{~d} s\right)\left(\int_{0}^{L} \Theta_{c}^{4} \mathrm{~d} s\right) \\
& +k_{t}\left[\left(\int_{0}^{L} \Theta_{c} \mathrm{~d} s\right)^{2}\left(\int_{0}^{L} \Theta_{c}^{4} \mathrm{~d} s\right)\right. \\
& \left.\left.-4\left(\int_{0}^{L} \Theta_{c} \mathrm{~d} s\right)\left(\int_{0}^{L} \Theta_{c}^{2} \mathrm{~d} s\right)\left(\int_{0}^{L} \Theta_{c}^{3} \mathrm{~d} s\right)\right]\right\} \\
& /\left(\int_{0}^{L} \Theta_{c}^{2} \mathrm{~d} s\right)\left\{\int_{0}^{L}\left[\Theta_{c}-\Theta_{c}(L)\right] \Theta_{c} \mathrm{~d} s\right\}
\end{aligned}
$$

Furthermore setting

$$
s=L \sigma, 0 \leqslant \sigma \leqslant 1
$$

and multiplying (28) by the factor $L^{3} / E I_{0}$ (where $E I_{0}$ is the bending rigidity at $\sigma=0$ ) we get a dimensionless counterpart for (28)

$$
\begin{aligned}
\beta_{m} & \geqslant \alpha_{w}+\frac{1}{3}\left\{\left[\int_{0}^{1}\left(\frac{E I}{E I_{0}}\right)\left(\frac{\mathrm{d} \Theta_{c}}{\mathrm{~d} \sigma}\right)^{2} \mathrm{~d} \sigma\right]\left(\int_{0}^{1} \Theta_{c}^{4} \mathrm{~d} \sigma\right)\right. \\
& +\left[\left(\int_{0}^{1} \beta_{e, o} \Theta_{c}^{4} \mathrm{~d} \sigma\right)\left(\int_{0}^{1} \Theta_{c}^{2} \mathrm{~d} \sigma\right)\right. \\
& \left.-\left(\int_{0}^{1} \beta_{e, o} \Theta_{c}^{2} \mathrm{~d} \sigma\right)\left(\int_{0}^{1} \Theta_{c}^{4} \mathrm{~d} \sigma\right)\right] \\
& -\gamma_{t}\left[\int_{0}^{1} \Theta_{c} \mathrm{~d} \sigma\right)^{2}\left(\int_{0}^{1} \Theta_{c}^{4} \mathrm{~d} \sigma\right) \\
& \left.\left.-4\left(\int_{0}^{1} \Theta_{c} \mathrm{~d} \sigma\right)\left(\int_{0}^{1} \Theta_{c}^{2} \mathrm{~d} \sigma\right)\left(\int_{0}^{1} \Theta_{c}^{3} \mathrm{~d} \sigma\right)\right]\right\}
\end{aligned}
$$

$$
/\left(\int_{0}^{1} \Theta_{c}^{2} \mathrm{~d} s\right)^{2}\left\{\int_{0}^{1}\left[\Theta_{c}-\Theta_{c}(1)\right] \Theta_{c} \mathrm{~d} \sigma\right\}=\alpha_{w}+q\left(\beta_{e}\right)
$$

where $\beta_{m}$ is the dimensionless weight of the contained fluid per unit length

$$
\beta_{m}=\frac{w_{m} L^{3}}{E I_{0}}=\rho_{m} g \frac{\pi D_{l}^{2}}{4} \frac{L^{3}}{E I_{0}}
$$

$\alpha_{w}$ is the dimensionless weight of the displaced fluid per unit length.

$$
\alpha_{w}=\frac{b_{w} L^{3}}{E I_{0}}=\rho_{w} g \frac{\pi D_{0}^{2}}{4} \frac{L^{3}}{E I_{0}}
$$

and $\beta_{e, o}$ is the dimensionless overhead effective weight

$$
\beta_{e, o}=\int_{0}^{1} \beta_{e} \mathrm{~d} \sigma=\frac{w_{e, o} L^{3}}{E I_{0}}
$$

Using the mode shape to evaluate the integrals in (28) or (30) we drive an inequality condition between the various parameters of the problem.

Inequality (30) can be transformed using equations (31) and (32) to one involving the densities of the fluids inside and outside the column

$$
\frac{\rho_{m}}{\rho_{w}} \geqslant\left(\frac{D_{0}}{D_{i}}\right)^{2}\left[1+\frac{q\left(\beta_{e}\right)}{\alpha_{w}}\right]
$$

Inequality (34) clearly shows that unstable behavior is observed when the ratio of the densities of the fluids inside and outside the column exceeds a certain value.

NUMERICAL IMPLEMENTATION BY FINITE ELEMENTS

The finite element method can be used to discretise the linearised equation (10). The discretised equation has the form $^{17}$

$$
\left(\left[K_{1}\right]+T_{t, a}\left[K_{2}\right]\right)\{\Theta\}=\{0\}
$$

$\left[K_{1}\right]$ is the sum of the elastic stiffness matrix and the geometric stiffness matrix due to effective weight. $\left[K_{2}\right]$ is a scaled geometric stiffness matrix due to top tension. The solution of (35) yields the critical top tension $\left(T_{t, a}\right)_{c}$ and the buckling mode vector $\left\{\Theta_{c}\right\}$. This vector can be used to evaluate the integrals in (28) or (30) numerically. To improve the approximation we use (10) to eliminate the bending stiffness term in (30). The inequality condition (36) becomes

$$
\begin{aligned}
& \beta_{m}-\alpha_{w} \geqslant+\frac{1}{3}\left[\left(\int_{0}^{1} \beta_{e, o} \Theta_{c}^{4} \mathrm{~d} \sigma\right)\right. \\
& \left.-4 \gamma_{t}\left(\int_{0}^{1} \Theta_{c} \mathrm{~d} \sigma\right)\left(\int_{0}^{1} \Theta_{c}^{3} \mathrm{~d} \sigma\right)-\delta_{c}\left(\int_{0}^{1} \Theta_{c}^{4} \mathrm{~d} \sigma\right)\right] \\
& /\left(\int_{0}^{1} \Theta_{c}^{2} \mathrm{~d} \sigma\right)\left\{\int_{0}^{1}\left[\Theta_{c}-\Theta_{c}(1)\right] \Theta_{c} \mathrm{~d} \sigma\right\}
\end{aligned}
$$

144 Applied Ocean Research, 1987, Vol. 9, No. 3 
The integrals in inequality (36) were evaluated numerically using the critical top tension and buckling mode vector of the finite element solution for a uniform column. The limiting value of $\beta_{m}-\alpha_{w}$ was calculated for various values of the dimensionless top spring stiffness. This value is plotted versus the column's dimensionless effective weight in Figs. 1 and 2 for hinged-hinged and clamped-clamped columns respectively. Results for hinged-clamped columns are within $1 \%$ of those for hinged-hinged columns for $\beta_{e} \gtrsim 500$. Similarly the results for clamped-hinged columns are within $1 \%$ of those for clamped-clamped columns for $\beta_{e} \gtrsim 500$. Figure 3 shows the results for all four combinations of end rotation conditions in the range $0 \leqslant \beta_{e} \leqslant 500$ for $\gamma_{t}=10^{5}$.

For columns with large $\beta_{e}$ equation (1) becomes singular. ${ }^{19}$ The slope of the centerline develops a region near the lower end in which most of the deformation is contained. This region for $\beta_{e} \rightarrow \infty$ becomes a boundary layer. The finite element model can yield accurate results if sufficient number of elements is used in this region. Obviously beyond some value of $\beta_{e}$ the required element length decreases (dimensionless problem) or the required number of elements increases (dimensional problem) prohibitively.

In the numerical implementation the results presented in Figs. 1 and 2 are a satisfactory approximation up to $\beta_{e} \cong$ 8000 . Beyond this point the finite element solution provides a lower bound to the exact solution.

\section{ASYMPTOTIC SOLUTION}

Uniform columns with movable top support, that is with zero top spring stiffness, attain an asymptotic behavior for $\beta_{e} \gtrsim 100 .^{19}$ Columns with nonmovable top support attain the same asymptotic behavior for $\beta_{e} \gtrsim 10^{51}$. The dimensionless governing linear differential equation in this case is

$$
\frac{\mathrm{d}^{2} \Theta}{\mathrm{d} \sigma^{2}}-\left[\delta-\beta_{e}(1-\sigma)\right] \Theta=0
$$

where $\delta$ is the dimensionless top tension

$$
\delta=\frac{T_{t, a} L^{3}}{E I_{0}}
$$

The asymptotic solution of the eigenvalue problem ${ }^{19}$ has critical dimensionless top tension

$$
\delta_{c}=\beta_{e}-x_{0} \beta^{2 / 3}
$$

and buckling mode shape

$$
\Theta_{c}(\sigma)=A i\left(x_{0}+\beta_{e}^{1 / 3} \sigma\right)
$$

$A i$ is the Airy function of the first kind and $x_{0}$ is the first root of $A i$ for columns with clamped lower end or the first route of $A i^{\prime}$ for columns with hinged lower end. These values are -2.338 and -1.018 respectively.

In the case of this asymptotic solution the inequality condition (36) becomes:

$$
\beta_{m}-\alpha_{w} \geqslant \frac{\int_{0}^{1} \Theta_{c}^{2} \Theta_{c}^{\prime 2} \mathrm{~d} \sigma}{\left(\int_{0}^{1} \Theta_{c}^{2} \mathrm{~d} \sigma\right)^{2}}
$$

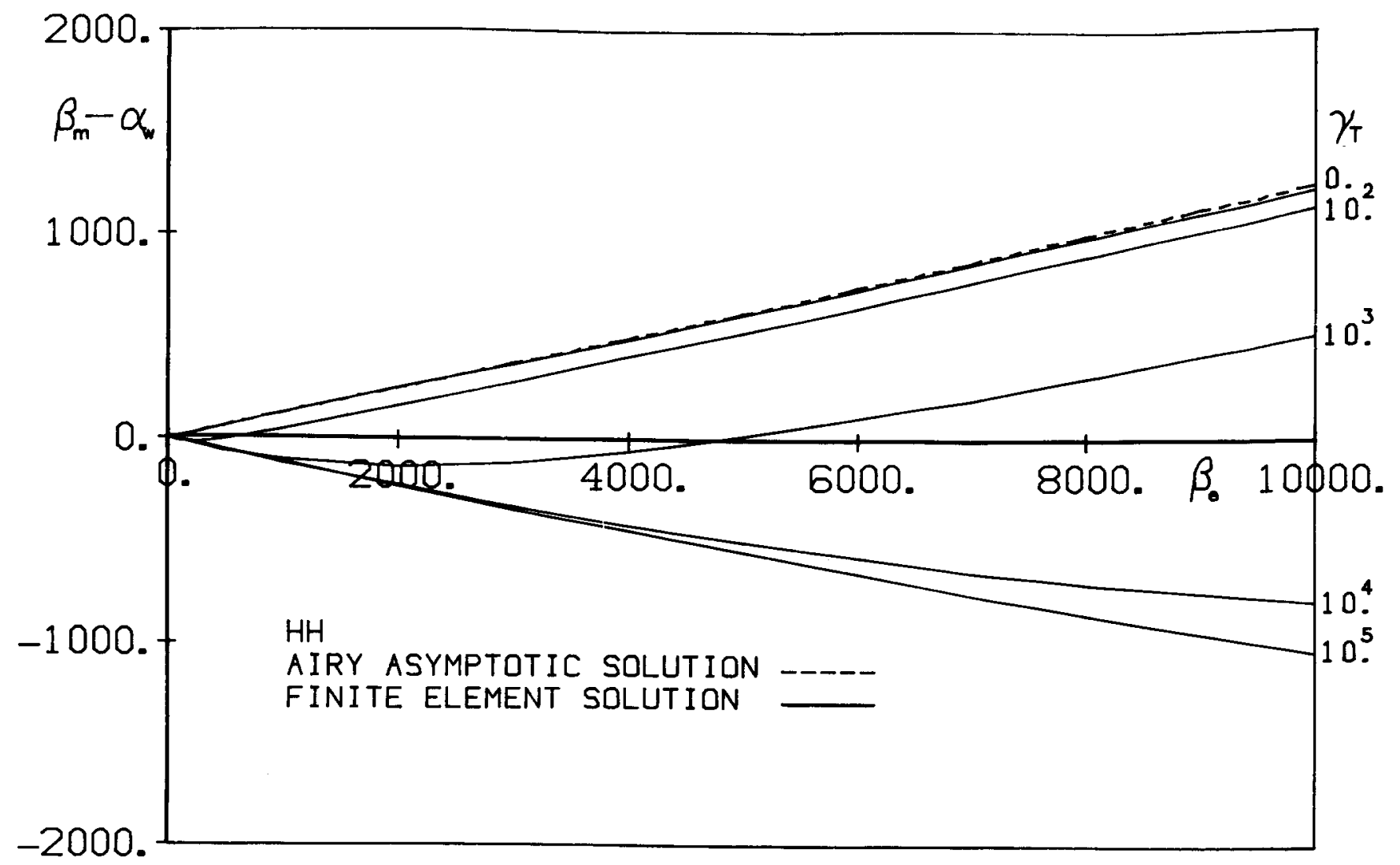

Figure 1. Limiting value of $\beta_{m}-\alpha_{w}$ for hinged-hinged columns 


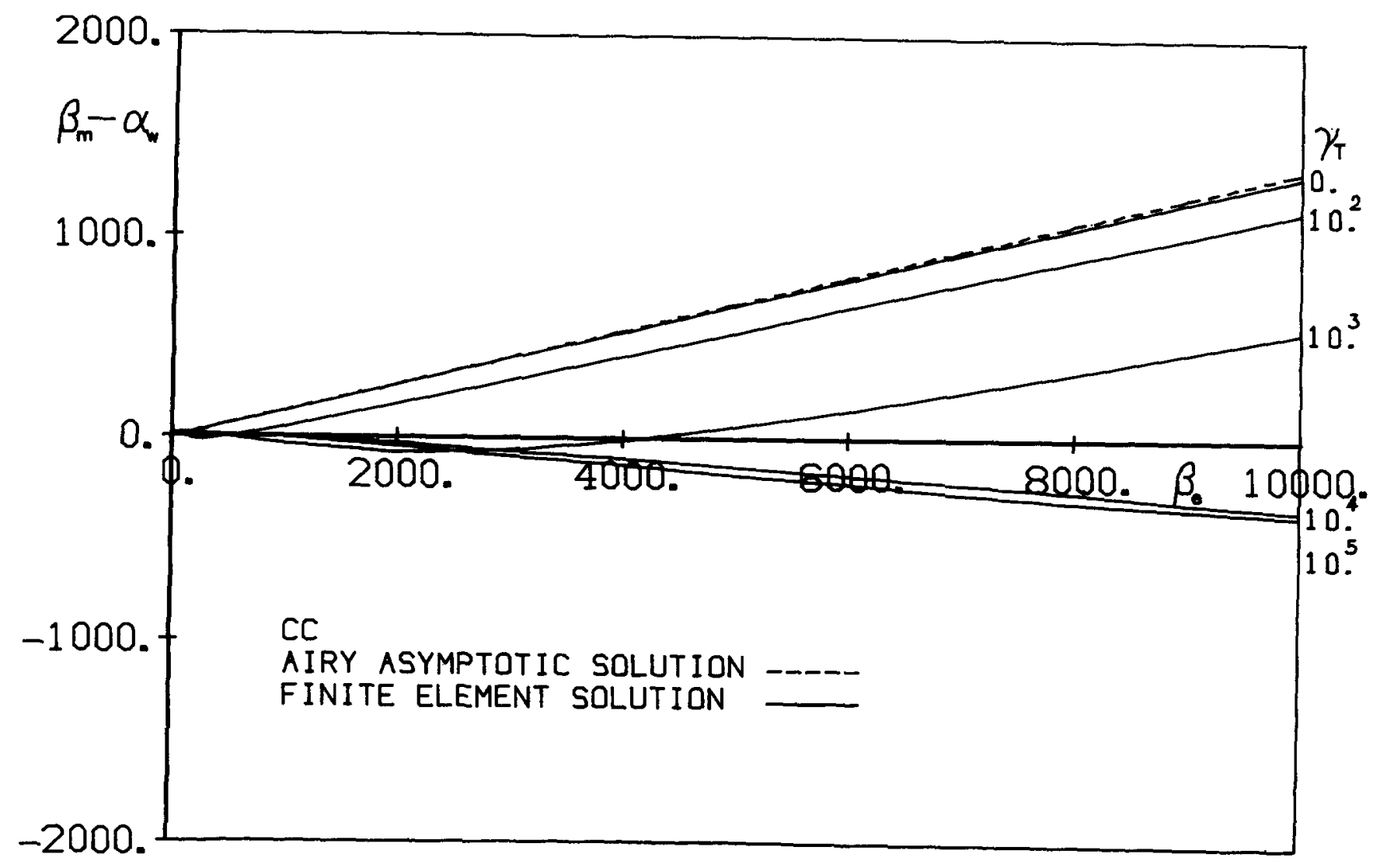

Figure 2. Limiting value of $\beta_{m}-\alpha_{w}$ for clamped-clamped columns

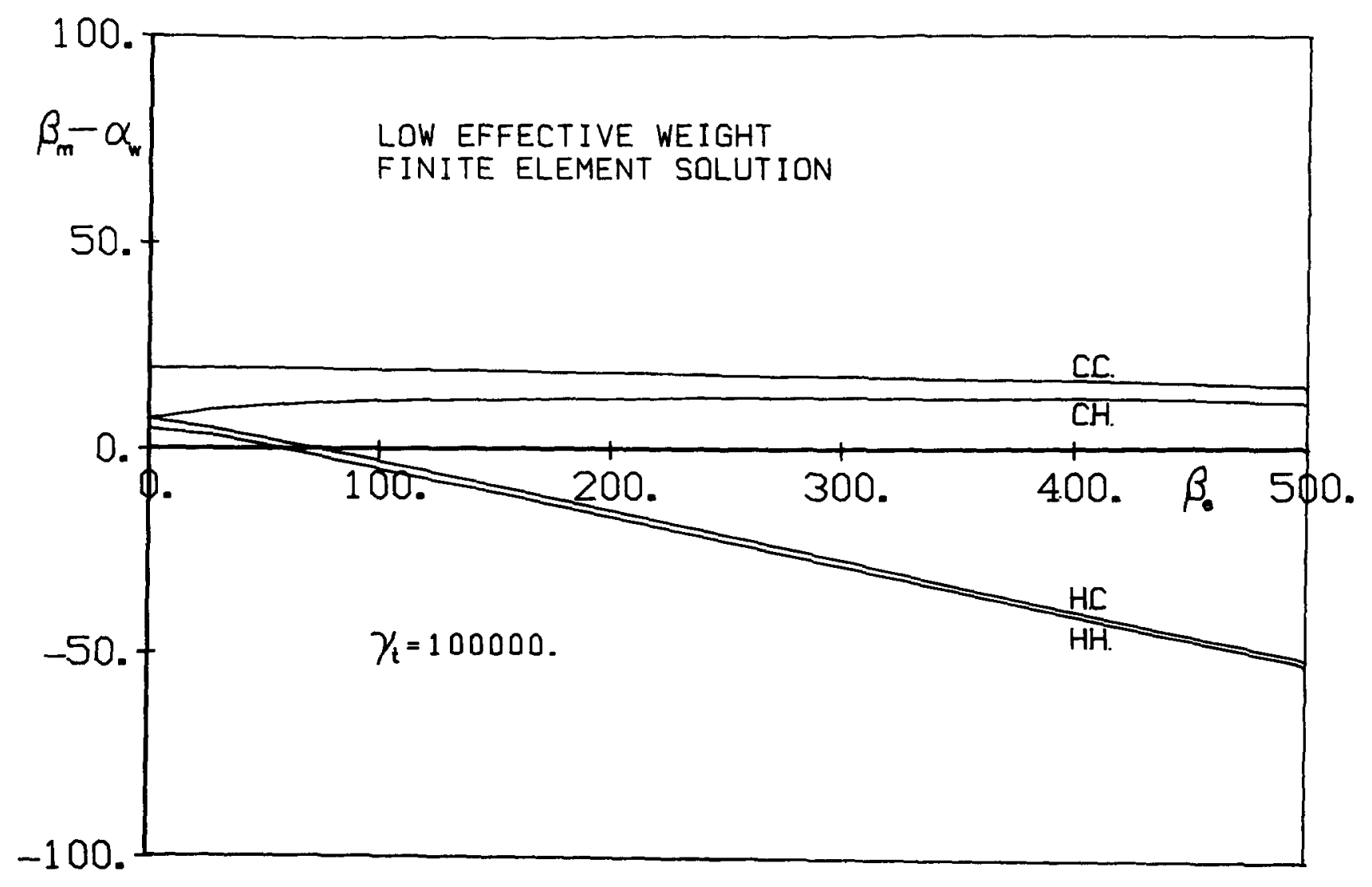

Figure 3. Limiting value of $\beta_{m}-\alpha_{w}$ for $0 \leqslant \beta_{e} \leqslant 500$ 


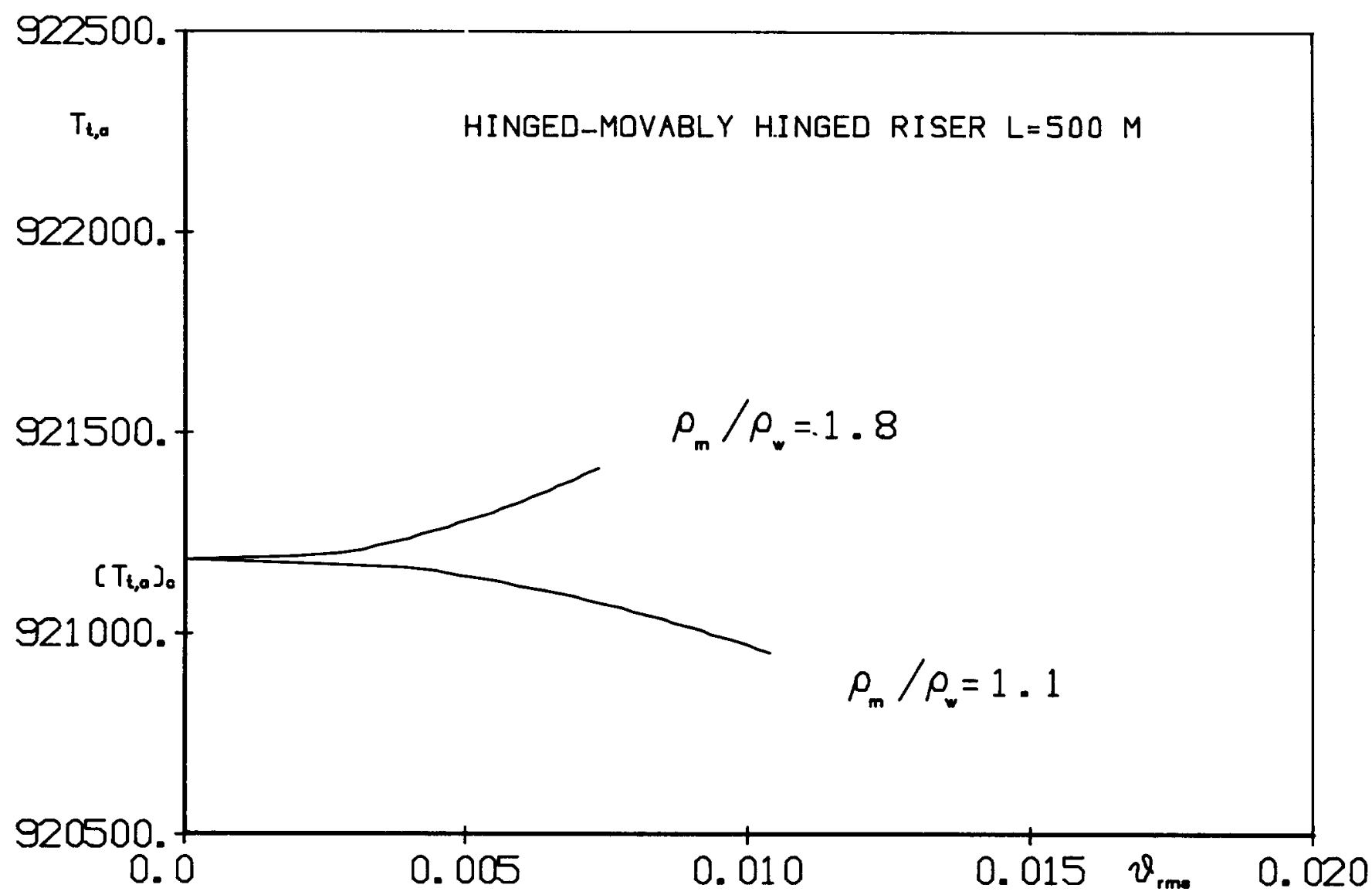

Figure 4. Secondary equilibrium paths of drilling riser

further using (40) in (41)

$$
\beta_{m}-\alpha_{w} \geqslant \frac{\int_{x_{0}}^{x_{0}+\beta_{e}^{1 / 3}} A i^{2}(x) A i^{\prime 2}(x) \mathrm{d} x}{\left(\int_{x_{0}}^{x_{0}+\beta_{e}^{1 / 3}} A i^{2}(x) \mathrm{d} x\right)^{2}}
$$

Care must be exercised in the numerical evaluation of the integrals in (42), because of the exponential decay of the Airy function of the first kind and its derivative. Namely, a Laguerre integration rule must be used in the interval $\left[2, x_{0}+\beta_{e}^{1 / 3}\right]$, while a Gauss integration rule may be used in the interval $\left[x_{0}, 2\right]$. The result is

$$
\beta_{m}-\alpha_{w} \geqslant \beta_{e} f\left(\beta_{e}\right)
$$

where $f\left(\beta_{e}\right)$ is a weakly increasing function of $\beta_{e}$. The product $\beta_{e} f\left(\beta_{e}\right)$ is indicated by the dashed curves in Figs. 1 and 2 .

\section{APPLICATION}

The results of this analysis were used to examine the initial postbuckling behavior of a $500 \mathrm{~m}$ long hinged-movably hinged marine drilling riser with inner diameter $0.46 \mathrm{~m}$ and outer diameter $0.5 \mathrm{~m}$, made of steel. The dimensionless effective weight is $\beta_{e}=1413$. Using the asymptotic solution the limiting value of $\beta_{m}-\alpha_{w}$ was found to be 312 .
The limiting value of the drilling mud density is $1366 \mathrm{~kg} /$ $\mathrm{m}^{3}$ or 1.33 times the density of salt water. Therefore it is well within the range of practical applications. Furthermore we must observe that this value will be even lower if the top of the riser is restrained horizontally.

Figure 4 shows the secondary equilibrium path for the riser of the above example for two different values of the drilling mud density, one below and one beyond the limiting value.

\section{CONCLUSIONS}

It was shown in this work that the initial postbuckling behavior of heavy tubular columns becomes unstable if the ratio of the densities of the fluids inside and outside the column exceeds a certain value. In such a case initial imperfections and lateral loads can affect the critical top tension of the column.

An inequality was derived for the difference between the dimensionless weight per unit length of the contained and the displaced fluid, $\beta_{m}-\alpha_{w}$, which if satisfied indicates unstable postbuckling behavior. The limiting value for this difference was calculated for a uniform column for various end conditions and plotted versus the column's dimensionless effective weight. Both a finite element solution and an asymptotic analytical solution for very long columns were implemented. This inequality was transformed to another one providing a limiting value for the ratio $\rho_{m} / \rho_{w}$.

Comparison of the results yielded by the finite element and the asymptotic solution for columns with movable top 
support indicates that the finite element solution provides an accurate approximation for $\beta_{e} \lesssim 8000$ and a lower bound beyond that for the limiting value of the difference $\beta_{m}-\alpha_{w}$.

For columns with movable top support the limiting value of $\beta_{m}-\alpha_{w}$ is almost proportional to the column's dimensionless effective weight $\beta_{e}$. The proportionality factor depends on the rotation condition at the lower end of the column and increases very slightly with $\beta_{e}$.

An elastic restraint of the column's top support has an appreciable effect on the limiting value of $\beta_{m}-\alpha_{w}$.

\section{ACKNOWLEDGEMENTS}

This work was supported by the Ocean Engineering Division of the American Bureau of Shipping.

\section{REFERENCES}

1 Koiter, W. T. On the Stability of Elastic Equilibrium, H. J. Paris, Amsterdam, 1945. English Translation AFFDL-TR-70-25

2 Brush, D. O. and Almroth, B. O. Buckling of Bars, Plates and Shells, MoGraw-Hill, New York, 1975

3 Greenhill, A. G. Determination of the greatest height consistent with stability that a vertical pole or mast can be made and of the greatest height to which a tree of given proportions can grow, Cambridge Philosophical Society Proceedings 1881, 4, 65

4 Willers, F. A. Das Knicken Schwerer Gestaenge, Zeitschrift fuer Angewandte Mathematik und Mechanik 1941, 21 (1), 43

5 Biezeno, C. B. and Koch, J. J. Note on the buckling of a vertically submerged tube, Applied Scientific Research, Sec. A 1943,1 (2), 131

6 Huang, T. and Dareing, D. W. Buckling and frequency of long vertical pipes, Journal of Engineering Mechanics Division, Proceedings ASCE 1967,95 (2), 167

7 Huang, T. and Dareing, D. W. Buckling and lateral vibration of drill pipe, Joumal of Engineering for Industry, Transactions ASME 1968, 90, 613

8 Bernitsas, M. M. and Kokkinis, T. Global static instability of risers Journal of Ship Research (in press)

9 Bernitsas, M. M. and Kokkinis, T. Buckling of risers in tension due to internal pressure: Nonmovable boundaries, 2nd International Offshore Mechanics and Arctic Engineering Symposium, Houston, Texas, February 1983 and ASME Transactions, Journal of Energy Resource Technology 1983, 105 (3)

10 Wang, C. Applied Elasticity, MoGraw-Hill, New York, 1953

11 Sewell, M. J. The static perturbation technique in buckling problems, Journal of Mechanics and Physics of Solids 1965, 13, 247

12 Croll, J. G. A. Continuum perturbation method in the branching analysis of conservative systems, International Journal of Mechanics and Science 1971, 13,605

13 Thompson, J. M. T. and Hunt, G. W. Comparative perturbation studies of the elastica, International Journal of Mechanics and Science 1969, 11, 999

14 Berkey, D. D. and Friedman, M. I. A perturbation technique applied to the buckling of a compressed elastica, Joumal of Computational and Applied Mathematics 1978, 4 (3), 213

15 Plaut, R. H. Postbuckling analysis of continuous elastic systems under multiple loads, Journal of Applied Mechanics, Trans. ASME 1979, 46 (2), 393

16 Wang, C. Y. Buckling and postbuckling of a long-hanging elastic column due to a bottom load, Journal of Applied Mechanics, Trans. ASME 1983, 50 (2), 311

17 Kokkinis, T. and Bernitsas, M. M. Initial postbuckling analysis of marine risers, Report to the American Bureau of Shipping, and Department of Naval Architecture and Marine Engineering, Ann Arbor, Publication No. 271, May 1983

18 Bolotin, V. V. Nonconservative Problems in the Theory of Elastic Stability, McMillan, New York, 1963

19 Bernitsas, M. M. and Kokkinis, T. Asymptotic analysis of column and riser stability boundaries, ASME Transactions, Journal of Applied Mechanics 1984, 51 (3), 560

\section{APPENDIX}

The centerline of the column at equilibrium is a two dimen. sional curve as shown in Fig. 5a. The effective tension and the effective weight of the column are given $b y^{17}$

$$
\begin{aligned}
& P_{e}(s)=T(s)-b_{w}(s)\left[h_{w}-z(s)\right]-w_{m}(s)\left[h_{m}-z(s)\right] \\
& w_{e}(s)=w_{s t}(s)+w_{m}(s)-b_{w}(s)-b_{m}(s)
\end{aligned}
$$

where $T$ is the actual tension in the column, $h_{m}$ and $h_{w}$ are the free surface ordinates of the fluids inside and outside the column, $w_{s t}$ is the weight of the tube per unit length and $b_{m}$ is additional buoyancy per unit length.

$$
\begin{aligned}
& \text { The equations of equilibrium are } \\
& \frac{\mathrm{d} P_{e}}{\mathrm{~d} s}-K Q-w_{e} \cos \Theta=0 \\
& K P_{e}+\frac{\mathrm{d} Q}{\mathrm{~d} s}+w_{e} \sin \Theta=0 \\
& \frac{\mathrm{d} M}{\mathrm{~d} s}+Q=0
\end{aligned}
$$

where $K$ is the curvature of the centerline, $Q$ the shear force and $M$ the bending moment. The equations are derived by cosidering the equilibrium of a differential element of the column in the deformed configuration. This is necessary in order to properly model the geometric nonlinearity without introducing any approximation at this point.

Use of the Euler-Bernoulii constitutive relationship for bending

$$
M=E I K=E I \frac{\mathrm{d} \Theta}{\mathrm{d} s}
$$

and elimination of variables between (A3), (A4), and (A5) yield the equation governing $\Theta$, the slope of the centerline.

$$
\begin{gathered}
\frac{\mathrm{d}}{\mathrm{d} s}\left\{\left[\frac{\mathrm{d}^{2}}{\mathrm{~d} s^{2}}\left(E I \frac{\mathrm{d} \Theta}{\mathrm{d} s}\right) / \frac{\mathrm{d} \Theta}{\mathrm{d} s}\right] \sin \Theta-\cos \Theta \frac{\mathrm{d}}{\mathrm{d} s}\left(E I \frac{\mathrm{d} \Theta}{\mathrm{d} s}\right)\right. \\
\left.-w_{e} \sin ^{2} \Theta / \frac{\mathrm{d} \Theta}{\mathrm{d} s}\right\}=0
\end{gathered}
$$

Figure $5 \mathrm{~b}$ shows the internal and external forces at the upper end of the column. $T_{t, a}$ is the applied tension at the top. $H_{t, s}$ is the horizontal linear spring force and $T_{t, f}$ is a fictitious fluidic tension which appears as a result of the method used to represent the fluid pressure forces. ${ }^{17} T_{t, f}$ is always tangent to the deformed centerline of the riser and therefore is a follower force. A follower force is nonconservative. Since $T_{t, f}$ is a resultant of the fluid pressure forces these are nonconservative too. The latter two are given by

$$
\begin{aligned}
& H_{t, s}=-k_{t} x(L)=-k_{t} \int_{0}^{L} \sin \Theta \mathrm{d} s \\
& T_{t, f}=b_{w}(L)\left[h_{w}-z(L)\right]-w_{m}(L)\left[h_{m}-z(L)\right] \\
& \text { Usually } h_{w} \cong h_{m} \cong L, \text { so that } \\
& T_{t, f}=\left[b_{w}(L)\right][L-z(L)]
\end{aligned}
$$




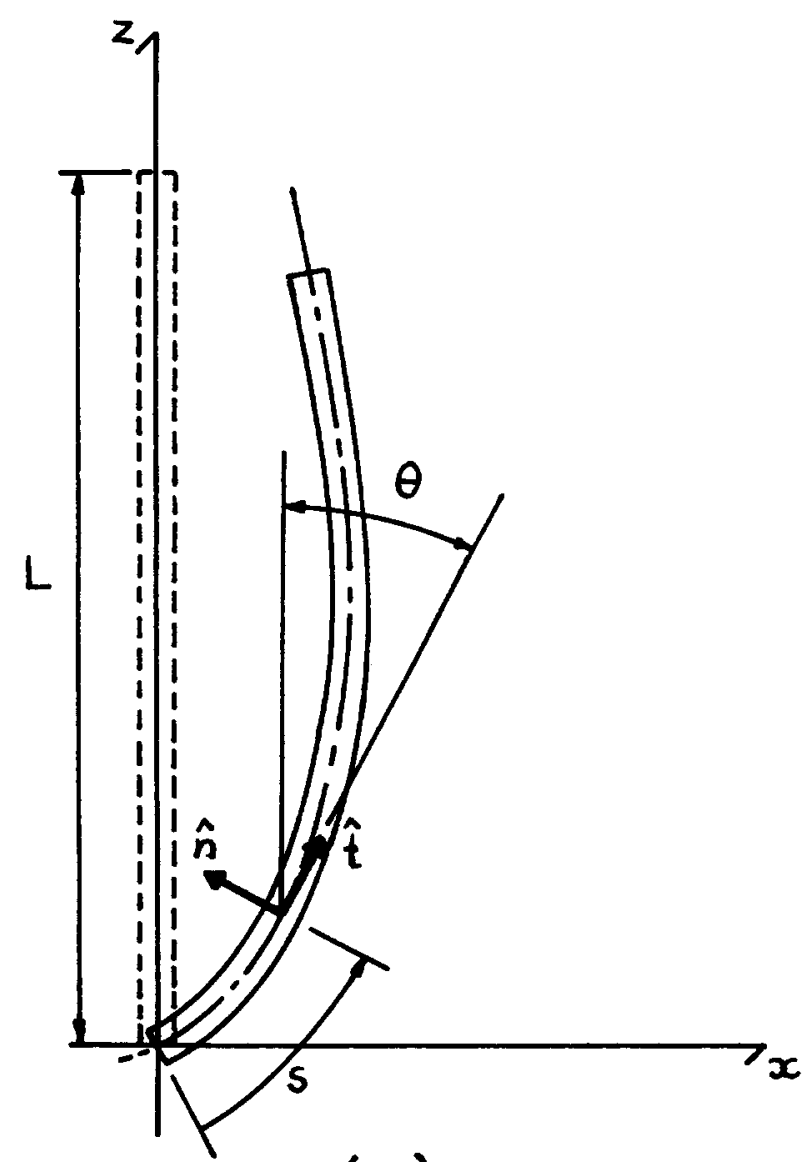

(a)

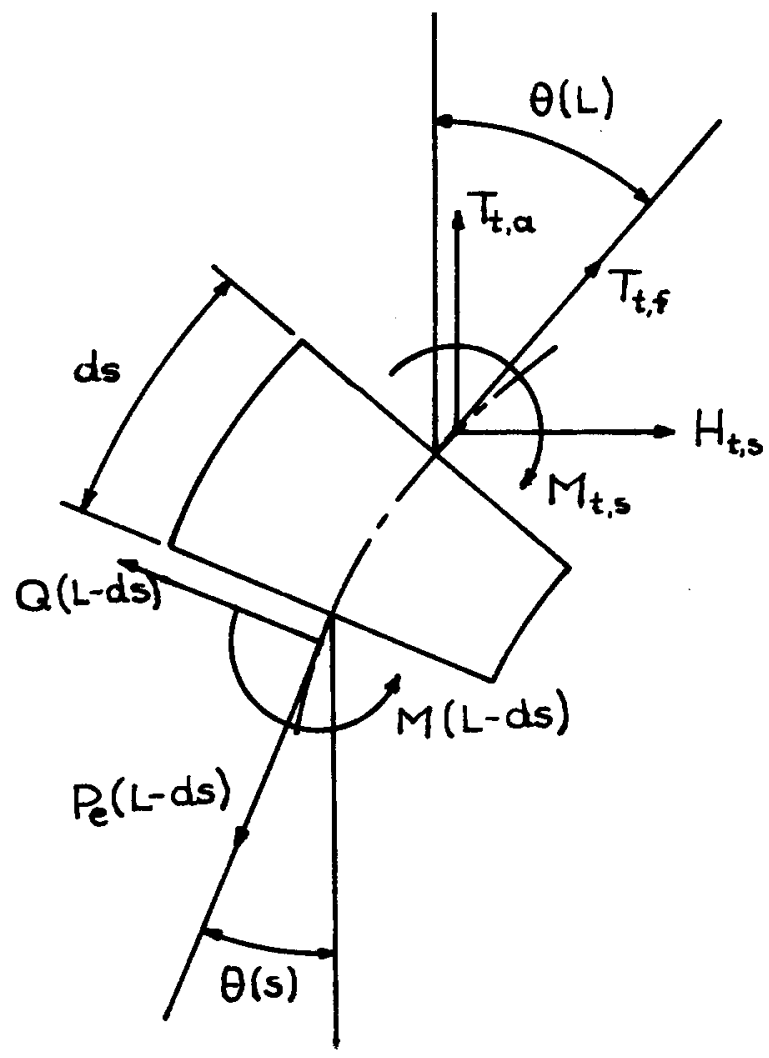

(b)

Figure 5. (a) Column equilibrium configuration. (b) Free body diagram of upper end element

The following are the equations of equilibrium of internal and external forces at the upper end of the column.

$$
\begin{gathered}
P_{e}(L)=T_{t, f}+T_{t, a} \cos \Theta(L)+H_{t, s} \sin \Theta(L) \\
Q(L)=-T_{t, a} \sin \Theta(L)+H_{t, s} \cos \Theta(L)
\end{gathered}
$$

Equation (A7) can be integrated twice and the two constants of integration can be found from (A11) and (A12). This yields the following equation

$$
\begin{aligned}
& \frac{\mathrm{d}}{\mathrm{d} s}\left(E I \frac{\mathrm{d} \Theta}{\mathrm{d} s}\right)-\left[-\int_{s}^{L} w_{e} \mathrm{~d} \sigma+T_{t, a}\right] \sin \Theta \\
& \quad-T_{t, f} \sin [\Theta-\Theta(L)]-k_{t} x(L) \cos \Theta=0
\end{aligned}
$$

